\title{
Editorial
}

Mario Plebani* and Martina Zaninotto

\section{Lot-to-lot variation: no longer a neglected issue}

https://doi.org/10.1515/cclm-2022-0128

Measurement is at the core of medical practice and laboratory testing is increasingly important for valuable clinical reasoning and patient management [1]. Much of the uncertainty in laboratory measurements cannot be completely eliminated because biological variation is always present even when analytical imprecision and bias have been minimised. Therefore, as stated by McCormack and Holmes "uncertainty in laboratory measurements is not a fixable problem but only a knowable problem" [2]. However, clinical laboratories have developed, improved over time and adopted into routine practice an arsenal of approaches, including validation/verification experiments and daily quality control procedures to ensure accurate and consistent results. In the last decade, valuable efforts have been made to ensure that analytical performance characteristics (APC) actually meet analytical performance specifications (APS) which are appropriate for a particular intended use of the specific measurand in clinical practice [3]. Monitoring measurement uncertainty (MU) and, in particular, withinlaboratory uncertainty $\left(\mathrm{U}_{\mathrm{RW}}\right)$ using internal quality control (iQC), plays a fundamental role in maintaining valuable APC, and many efforts have been and are being made to improve $\mathrm{iQC}$, including the integration of traditional programmes with patient-based real-time quality control [4]. However, limited attention has been paid to within-lot and between-reagent lot variations, which can strongly affect the analytical and clinical reliability of laboratory results. In this issue of the Journal, two papers provide new and important information for clinical laboratory professionals on the issue of reagent lot variation. The first paper highlights some recent examples of changes in analytical performances due to significant drift/shift that have resulted in clinical harm, involving critical laboratory tests such as high-sensitivity troponin $\mathrm{T}$, prostate-specific antigen, gentamicin and insulin-like growth factor-1 assays [5]. The

*Corresponding author: Mario Plebani, Honorary Professor, Clinical Biochemistry and Clinical Molecular Biology, University of Padova, Padua, Italy; and QI.LAB.MED, Spin-off of the University of Padova, Padua, Italy, E-mail: mario.plebani@unipd.it. https://orcid.org/ 0000-0002-0270-1711

Martina Zaninotto, QI.LAB.MED, Spin-off of the University of Padova, Padua, Italy authors emphasise that the "drift/shift in results was first detected by clinicians and not by routine laboratory procedures and quality systems". In this paper, a series of current challenges with lot-to-lot verification are recognised, including the lack of an industry-wide definition of a reagent lot, the range of concentration and number of samples used and their commutability, as well as the definition of acceptable analytical performance. In addition, the authors make interesting suggestions and proposals for potentially overcoming the reagent lot-to-lot drift/shift problems, including the request to manufacturers to improve the transparency of in-house processes and definition of a reagent lot, the validation and verification processes and the performance specifications adopted for accepting a lot; they correctly emphasise that regulatory changes - particularly the new European Union Medical Device Regulation - may catalyse the improvement process. Further suggestions include the need to use commutable material in external quality assessment (EQA)/proficiency testing (PT) programmes, the role of patient-based quality control and, finally, the need to harmonise acceptance criteria in reagent lot verification. It should be considered a stroke of luck that the second paper published in this issue of the Journal and prepared by a Working Group of the European Federation of Clinical Chemistry and Laboratory Medicine (EFLM) adds further insights into the topic. In fact, it provides a guide for balancing the lot-to-lot acceptance criteria with the actual utility of a laboratory test in clinical pathways and decision making [6]. The authors summarise the current shortcomings of currently available guidelines on lot-to-lot variation, including the Clinical and Laboratory Standards Institute (CLSI) [7] EP26A and EP-31-A-IR [8], and postulate an interesting model for allocating a part of allowable $U_{R W}$ to between-reagent lot variation, based on the need for long-term consistency of the measurement variability for a specific measurand. The allocation should control the ratio between short-term and long-term variation and provide guidance to clinical laboratories when to reject or correct certain variations due to reagent lot. The establishment of allowable between-lot variation is based on some premises: (a) the knowledge of within-subject biological variation, the source of information being the EFLM database (https://biologicalvariation.eu/); (b) the number of results from a single patient within the period of use of the reagent lot, based on intended use and/or professional 
opinion; (c) the use of an equation (4) which complies with the allowable MU. From a practical point-of view, the detailed approach described in the document seems to be of particular value to check the lot-to-lot variation in a statistically sound and simultaneously practical way. In particular, the paragraph "Mathematical evaluation of allowable between-reagent lot variation" provides an objective generally applicable procedure to verify the consistency of the results obtained with different reagent lots for a specific test. In the calculation described, in addition to the uncertainty components under conditions of laboratory precision $\left(\mathrm{u}_{\mathrm{RW}}\right)$, the number of measurements of a patient within a single reagent lot was also taken into account [9]. These additional data, arbitrarily set to six in the given equation, allow for a minimum budget for between-lot reagent variation, as underlined by the authors. Furthermore, the $\mathrm{n}$ value in the equation that represents a typical number of results for a single patient within the period of use of the reagent, should be based on the intended use of the test, the clinical opinion and the protocol adopted in each specific clinical setting: indeed, this information can be easily derived from the laboratory information system [10]. The suggested approach can therefore allow systematic monitoring of both the amount of variation due to changes in reagent lots and longterm drifts. However, there is still a need for manufacturers to compare the performance of a new reagent batch to higherorder reference material and/or method in the selected metrological traceability chain, using commutable materials and provide transparent and typical performance information to users. Both papers should therefore be very welcome by laboratory professionals as they draw attention to reagent lot variations to avoid drifts/shifts in analytical performances that may carry the risk of patient harm. In addition, they provide new and interesting information and suggestions for dealing with and monitoring between-lot variation in routine practice and illustrate that analytical quality is a never-ending process. We believe that these papers not only deserve major interest and confident use by laboratory professionals, but they must also stimulate further research in the field of quality control in laboratory medicine.
Research funding: None declared.

Author contributions: All authors have accepted responsibility for the entire content of this manuscript and approved its submission.

Competing interests: Authors state no conflict of interest.

\section{References}

1. Plebani M. Charting the course of medical laboratories in a changing environment. Clin Chim Acta 2002; 319:87-100.

2. McCormack JP, Holmes DT. Your results may vary: the imprecision of medical measurements. BMJ 2020;368:m149.

3. Panteghini M, Ceriotti F, Jones G, Oosterhuis W, Plebani M, Sandberg S, et al. Strategies to define performance specifications in laboratory medicine: 3 years on from the Milan Strategic Conference. Clin Chem Lab Med 2017;55: 1849-56.

4. van Rossum HH, Bietenbeck A, Cervinski MA, Katayev A, Loh TP, Badrick TC. Benefits, limitations, and controversies on patientbased real-time quality control (PBRTQC) and the evidence behind the practice. Clin Chem Lab Med 2021;59:1213-20.

5. Loh TP, Sandberg S, Horvath AR. Lot-to-lot reagent verification: challenges and possible solutions. Clin Chem Lab Med 2022;60: 675-80.

6. van Schrojentein Lantman M, Cubukcu HC, Panteghini M, Bernabeu AF, Milinkovic N, Mesko BP, et al. An approach for determining allowable between reagent lot variation. Clin Chem Lab Med 2022;60:681-8.

7. Clinical and Laboratory Standards Institute. EP26A: user evaluation of between-reagent lot variation; 2013. Available from: https://clsi.org/standards/products/method-evaluation/ documents/ep/26/.

8. Clinical and laboratory Standards Institute EP-31A-IR. Verification of comparability of patient results within one health care system; Approved Guideline (Interim Revision); 2012.

9. Plebani M, Sciacovelli L, Bernardi D, Aita A, Antonelli G, Padoan A. What information on measurement uncertainty should be communicated to clinicians and how? Clin Biochem 2018;57: 18-22.

10. Pelloso M, Basso D, Padoan A, Fogar P, Plebani M. Computerbased-limited and personalised education management maximise appropriateness of vitamin D, vitamin B12 and folate retesting. J Clin Pathol 2016;69:777-83. 